\title{
Comparison of traditional cloud-point extraction and on-line flow-injection cloud-point extraction with a chemiluminescence method using benzo[a]pyrene as a marker
}

Received: 30 September 2005 / Revised: 2 November 2005 / Accepted: 4 November 2005 / Published online: 22 December 2005 (C) Springer-Verlag 2005

\begin{abstract}
In this work, using benzo(a)pyrene $(\mathrm{BaP})$ as marker, the analytical merits of on-line flow-injection cloud-point extraction (FI CPE), including preconcentration factor, extraction efficiency, sample throughput, and analysis time were evaluated by use of peroxyoxalate chemiluminescence (CL) detection. Moreover, by detailed discussion of several preconcentration conditions for traditional and on-line FI CPE the advantages of on-line FI CPE became conspicuously apparent. When coupled with separation techniques such as high-performance liquid chromatography (HPLC) or capillary electrophoresis (CE), on-line FI CPE-CL has much potential for analysis of low concentrations of polycyclic aromatic hydrocarbons (PAH) in environmental samples.
\end{abstract}

Keywords On-line flow-injection cloud-point extraction · Traditional cloud-point extraction - Chemiluminescence . $\operatorname{Benzo}(a)$ pyrene

\section{Introduction}

Aqueous solutions of almost all non-ionic or zwitterionic surfactants become turbid when heated to a temperature referred to as the cloud point. Addition of salting-out agents has proven to be another effective means of inducing the clouding phenomenon $[1,2]$. When cloud-point extraction (CPE) is performed in aqueous solutions,

\footnotetext{
G. Q. Song · J.-M. Lin $(\bowtie)$

State Key Laboratory of Environmental Chemistry and Ecotoxicology, Research Center for Eco-Environmental Sciences, Chinese Academy of Sciences,

P.O. Box 2871 Beijing, 100085, People's Republic of China e-mail: jmlin@mail.rcees.ac.cn

Tel.: 86-01-62841953

Fax: 86-01-62841953

C. $\mathrm{Lu} \cdot \mathrm{K}$. Hayakawa

Graduate School of Natural Science and Technology,

Faculty of Pharmaceutical Sciences, Kanazawa University,

Kakuma-machi,

Kanazawa, 920-1192, Japan
}

hydrophobic compounds without charges repel water, resulting in the concentration of these compounds in the small volume of the surfactant-rich phase, whereas hydrophilic components remain in the residual aqueous solution [3]. The phase separation is reversible - when the mixture is cooled to a temperature below cloud point the two phases merge to form a single phase again.

CPE has been extensively used for preconcentration/ extraction of hydrophobic species from aqueous solutions $[4,5]$. As an alternative to other preconcentration techniques, CPE uses a small amount of nonflammable and nonvolatile surfactant, instead of organic solvents, in solvent extractions such as liquid-liquid extraction [6]. CPE involves a series of fussy procedures, however, including equilibration, centrifugation, and removal of the surfactant-rich phase, resulting in poor reproducibility and low preconcentration factor. Widespread application of $\mathrm{CPE}$ is, therefore, very limited.

Flow-injection analysis (FIA) is, owing to its versatility, simplicity, and low instrumental cost, a powerful tool for automatic rapid sample pretreatment and nonchromatographic separation before determination of the analyte by a variety of detection methods [7]. On-line combination of CPE with FIA has been shown to be a promising means of analysis because of enhanced sensitivity and reproducibility, efficient removal of matrices, and high extraction efficiency. In on-line FI CPE, the equilibration and centrifugation procedures are avoided, making the entire extraction process simpler and the analysis time shorter. More importantly, the surfactant-rich phase containing the analytes can be easily removed by on-line elution rather than use of a pipette. A larger preconcentration factor can therefore be achieved by use of lower concentrations of surfactant. In recent years, on-line combination of CPE with FIA has attracted much attention. For example, Cordero and co-workers [8] were the first to recognize the advantages of combining CPE with FIA, but the cloudpoint methodology was still performed off-line in their experiments. The ability to perform CPE on-line in an FIA system was been reported for the first time by Huie [9]. Paleologos [10] has reported the determination of metal 
species by on-line combination of CPE with FIA coupled with chemiluminescence detection. Analysis of mercury by on-line FI CPE has been reported by Garrido [11].

In this work we have systemically evaluated the analytical performance of on-line FI CPE, including preconcentration factor, extraction efficiency, analysis time, and technical aspects. To overcome the strong UV-visible absorption interference from nonionic surfactants and to enhance detection sensitivity, a relatively stable peroxyoxalate chemiluminescence (CL) system was used for detection $[12,13]$. We investigated $16 \mathrm{PAH}$ named as priority pollutants by the EPA and found that benzo $(a)$ pyrene $(\mathrm{BaP})$ resulted in the highest $\mathrm{CL}$ intensity. We therefore used $\mathrm{BaP}$ as a marker.

\section{Experimental}

Apparatus

The FIA manifold for on-line FI CPE is illustrated in Fig. 1. It consisted of two peristaltic pumps (SJ-1211; Atto,
Tokyo, Japan), a six-channel injection valve (Sanuki, Japan) and a man-made CPE collection column (glass, $3.0 \mathrm{~cm}$ long, $0.3 \mathrm{~cm}$ i.d. packed with cotton). The CL intensity signal was recorded as peak height. The signal data were obtained using a F-2500 fluorescence spectrophotometer (Hitachi, Tokyo, Japan) at the maximum emission wavelength, $415 \mathrm{~nm}$. The excitation lamp was off and the emission slit width was fully opened to $20 \mathrm{~nm}$ during CL intensity recording. In traditional CPE a centrifuge (LG10-2.4A, Beijing Medical Centrifuge Factory, Beijing, China) was also used to hasten complete phase separation.

\section{Chemicals}

Analytical grade $\mathrm{BaP}$, benzo[ $k]$ fluoranthene $(\mathrm{BkF})$, and benzo[ghi]perylene (Bper) were obtained from Sigma (St Louis, MO, USA). Triton X-114 and acetonitrile (HPLC grade) were obtained from Acros (Geel, Belgium) and Siyou Biologic and Technic Company (Tianjing, China), respectively. Sodium sulfate and $30 \%$ hydrogen peroxide
Fig. 1 Schematic diagram of the on-line FI CPE system. $P_{l}$ and $P_{2}$, peristaltic pumps; $R_{l}$, sample solution $(\mathrm{BaP}$ standard and Triton $\mathrm{X}-114) ; R_{2}$, saltingout agent $\mathrm{Na}_{2} \mathrm{SO}_{4} ; R_{3}$, acetonitrile; $C$, collection column (effective length $1.5 \mathrm{~cm}$, i.d. $3 \mathrm{~mm}) ; W_{1}$ and $W_{2}$, waste

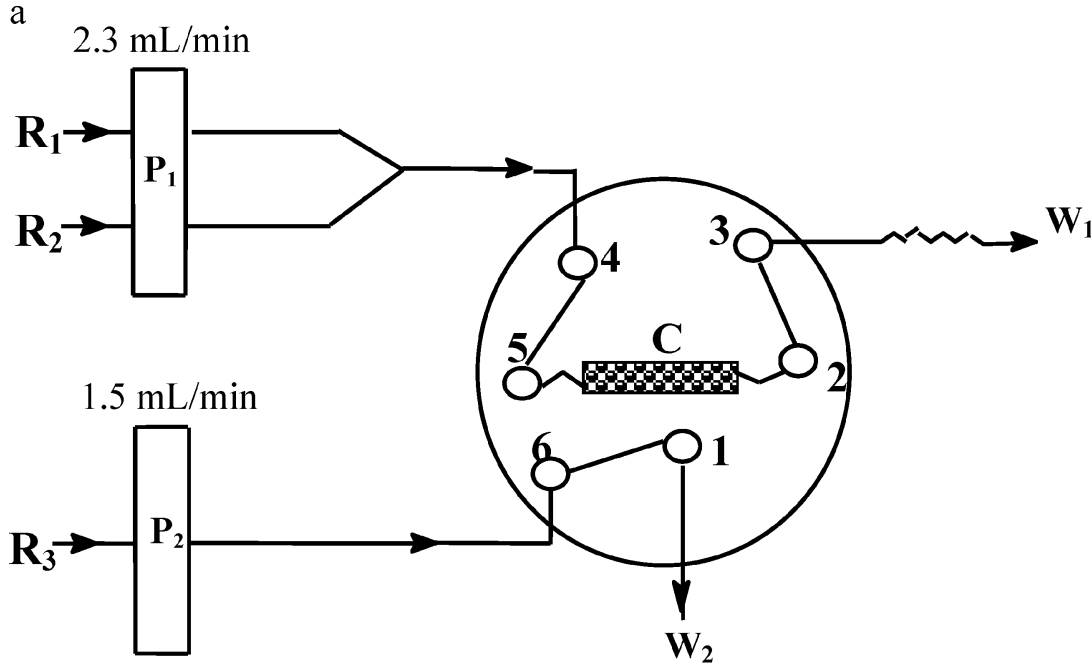

b

$2.3 \mathrm{~mL} / \mathrm{min}$

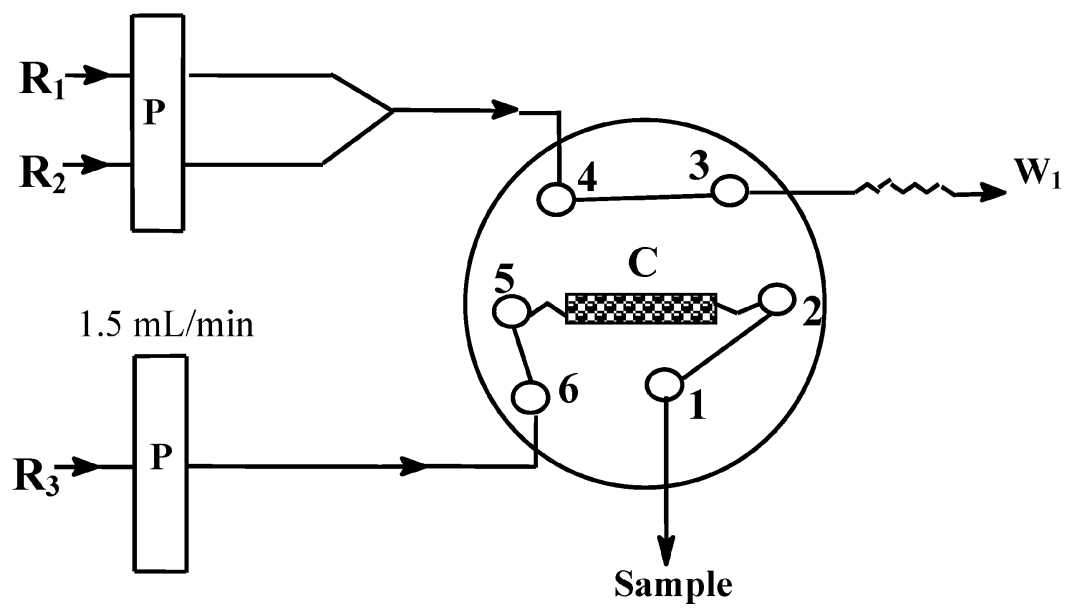


$\left(\mathrm{H}_{2} \mathrm{O}_{2}\right)$ were obtained from Beijing Chemical Company (China). Bis(2,4,6-trichlorophenyl)oxalate (TCPO) was purchased from Fluka (Lake Ronkonkoma, NY, USA). Water was deionized by means of a Milli-Q purification system with a $0.2 \mu \mathrm{m}$ fiber filter (Barnstead, USA). Cotton was commercial absorbent cotton and made of pure cellulose (Beijing Filter Company, Beijing, China).

A stock solution $\left(250 \mu \mathrm{g} \mathrm{mL}^{-1}\right)$ of BaP was prepared by dissolving the standard in acetonitrile and working solution was prepared by dilution with Triton X-114 solution. Triton $\mathrm{X}-114$ solutions were prepared by dissolving appropriate amounts of Triton X-114 in water. Sodium sulfate solution was prepared by dissolving the salt in water and was filtered through a $0.45 \mu \mathrm{m}$ pore-size fiber filter before use. $\mathrm{H}_{2} \mathrm{O}_{2}$ working solution was freshly prepared by diluting $30 \% \mathrm{H}_{2} \mathrm{O}_{2}$ with acetonitrile. TCPO working solutions were prepared by dissolving appropriate amounts of TCPO in acetonitrile.

\section{Procedures}

\section{Traditional cloud point extraction}

Typically, a traditional CPE experiment was performed as follows. Appropriate amounts of aqueous solutions containing the analyte in the presence of Triton X-114 were prepared. Cloud-point separation was initiated by addition of appropriate amounts of sodium sulfate. The final volume of the bulk solution was $25 \mathrm{~mL}$. The concentrations of sodium sulfate and Triton X-114 were $0.6 \mathrm{~mol} \mathrm{~L}^{-1}$ and $1.0 \%(v / v)$, respectively. The sample solution was left to equilibrate for $10 \mathrm{~min}$ and then centrifuged at $3500 \mathrm{rpm}$ for 20 min to achieve complete separation of the two phases. The interface between the top surfactant-rich phase and the bottom aqueous phase was very sharp. The surfactant-rich phase was carefully transferred to a glass tube by means of a pipette. Appropriate amounts of the surfactant-rich phase, TCPO, and $\mathrm{H}_{2} \mathrm{O}_{2}$ were sequentially added to a $3.0-\mathrm{mL}$ quartz cell for CL measurements with the wavelength of the emission monochromator set to $415 \mathrm{~nm}$.

\section{On-line FI cloud point extraction}

On-line FI CPE preconcentration of $\mathrm{BaP}$ was performed as follows. In the extraction stage (Fig. 1a), sample solution containing $0.05 \mu \mathrm{g} \mathrm{mL}^{-1} \mathrm{BaP}$ and $0.05 \%(v / v)$ Triton X114 was first loaded into an FIA manifold via channel $R_{1}$. A flow rate of $2.3 \mathrm{~mL} \mathrm{~min}{ }^{-1}$ and a loading time of $90 \mathrm{~s}$ were used for the sample solution, resulting in loading of $3.45 \mathrm{~mL}$ sample solution. The sample solution from $\mathrm{R}_{1}$ merged with the salt solution from $\mathrm{R}_{2}$ to induce separation of the sample solution into two distinct phases - a surfactant-rich phase containing molecules of $\mathrm{BaP}$ solubilized within surfactant aggregates and an aqueous phase containing the salt, surfactant monomers, and other watersoluble components of the sample solution. The mixture then passed through the collection column, which enabled the surfactant-rich phase to be trapped inside the column while the aqueous phase passed through the column. When sample loading was complete the injection valve was switched to the position shown in Fig. 1b, the elution solvent passed through the column at the rate of $1.5 \mathrm{~mL} \mathrm{~min}^{-1}$, carrying the $\mathrm{BaP}$ and surfactant molecules into a glass tube. The amount of $\mathrm{BaP}$ in the surfactant-rich phase was measured by the CL method used with traditional cloud point extraction. The theoretical preconcentration factor was calculated as the ratio of the volume of the original solution to the volume of the surfactant-rich phase, which simultaneously varies with the amount of the surfactant $[4$, 14]. In this work, the preconcentration factor (PF) is defined by the equation: $\mathrm{PF}=\left(A_{2}-A_{1}\right) / A_{0}$, where $A_{0}$ is the CL intensity of the original sample solution (without preconcentration), $A_{2}$ and $A_{1}$ denote the CL intensities of the $\mathrm{BaP}$ solution after preconcentration and the blank, respectively, and $A_{0}$ and $A_{2}$ are linearly dependent on the concentration of $\mathrm{BaP}$. The emission wavelength was set at the peak maximum for BaP, i.e. $415 \mathrm{~nm}$.

In on-line FI CPE, $A_{2}$ and $A_{1}$, respectively, were determined by collecting the concentrated $\mathrm{BaP}$ and the blank at sample-output end 1 (Fig. 1b) between 8.8 and $35.2 \mathrm{~s}$ after loading of sample solution into the FIA system. For the determination of $A_{0}$, the same volume of sample solution was also collected but without the preconcentration step (without addition of salt and without the presence of the collection column).

It is well known that micelles and some inorganic cations can increase the CL intensity of analytes in the peroxyoxalate CL system $[15,16]$. With this system, therefore, to evaluate enhancement of the signal because of concentration of the analyte rather than the enhancement because of the effect of the cloud-point phenomenon itself, we deducted the CL signal intensity resulting from micellar and salt enhancements by performing a blank experiment (i.e. performing CPE under the optimum conditions but without $\mathrm{BaP}$ solution).

\section{Results and discussion}

\section{Selection of chemiluminescence detection}

It is well known that the concentration of the surfactant containing the analytes is greatly increased after CPE, resulting in strong UV-visible and fluorescence background absorption. Application of spectrophotometry and fluorescence detection for CPE was therefore restricted. Chemiluminescence detection does not require excitation source, however, and thus can overcome these disadvantages. It has been successfully used as a detection technique after $\mathrm{CPE}[10,17]$. In this work the TCPO- $\mathrm{H}_{2} \mathrm{O}_{2} \mathrm{CL}$ system was used for analysis of $\mathrm{BaP}$ after CPE. The method is based on oxidation of aryl oxalate esters by $\mathrm{H}_{2} \mathrm{O}_{2}$ in the presence of a suitable fluorescent species. The concentrations of TCPO and $\mathrm{H}_{2} \mathrm{O}_{2}$ were optimized, and $2.5 \mathrm{mmol} \mathrm{L}^{-1}$ TCPO and $3.0 \mathrm{~mol} \mathrm{~L}^{-1} \mathrm{H}_{2} \mathrm{O}_{2}$ were selected for the entire experiment. 


\section{Effect of Triton X-114 concentration}

The efficiency of CPE is very dependent on the concentration of surfactant. It is known that Triton X-114 has a low cloud-point temperature, results in a surfactant rich phase of high density, is commercial available at low cost, and has no electroactive groups in its molecule [18]. Triton $\mathrm{X}-114$ is, therefore, often used in CPE to form the surfactant-rich phase. In this work the CL intensity of online FI CPE as a function of Triton X-114 concentration was investigated. It was found that the maximum CL intensity was obtained for $0.05 \%(v / v)$ Triton X-114. Larger concentrations of the surfactant led to the decreasing CL intensity, presumably because larger concentrations increase the volume of the surfactant-rich phase within the collection column, resulting in dilution of the extracted sample. The effect of the concentration of Triton X-114 in traditional CPE was also examined. It was proved that the highest CL intensity was at $0.8 \%(v / v)$. Throughput this study, however, the traditional CPE system was operated with $1.0 \%(v / v)$ Triton X-114 because lower Triton X-114 concentrations resulted in difficulty collecting the surfactant-rich phase and, subsequently, unacceptable experimental error.

The theoretical preconcentration factor is defined as the ratio of the volume of the original aqueous phase to that of the surfactant-rich phase after the phase-separation step [4, 14]. The volume of the surfactant-rich phase is proportional to the surfactant concentration in the original aqueous solution, and the preconcentration factor increases with decreasing volume of the surfactant-rich phase. From these results we can see that the optimum Triton X-114 concentration for on-line FI CPE was much lower than that for traditional CPE, which resulted in a higher theoretical preconcentration factor. In traditional CPE it is necessary to maintain the surfactant-rich volume sufficient for removal of the concentrated samples whereas in on-line FI CPE without the removal step the preconcentration factor can be improved by reducing the original surfactant concentration. Compared with traditional CPE, this characteristic of online FI CPE is one of the most significant advantages.

\section{Effect of salting-out agents}

On the basis of the cloud point phenomenon [1, 2] an appropriate salting-out agent a acting as a cloud-point temperature $\left(T_{\mathrm{c}}\right)$-depressing electrolyte can be used for preconcentration of hydrophobic species from an aqueous phase into a non-ionic surfactant phase (Triton X-114). Salting-out agents $\mathrm{Na}_{2} \mathrm{SO}_{4}, \mathrm{NaCl}, \mathrm{K}_{3} \mathrm{PO}_{4}$, and $\mathrm{NaOH}$ $\left(0.8 \mathrm{~mol} \mathrm{~L}^{-1}\right.$ each) were compared for their ability to yield the highest CL intensity for detection of CPE concentrated BaP. It was found that $\mathrm{Na}_{2} \mathrm{SO}_{4}$ resulted in the highest CL intensity. The effect of salting-out agent concentration on $\mathrm{CL}$ intensity was investigated. It was found that an increase in $\mathrm{Na}_{2} \mathrm{SO}_{4}$ concentration caused a concomitant increase of $\mathrm{CL}$ intensity. When the $\mathrm{Na}_{2} \mathrm{SO}_{4}$ concentration was more than $0.8 \mathrm{~mol} \mathrm{~L}^{-1}$, crystal particles appeared and occasional blockage of FIA channels occurred. The optimum salt concentration of $0.8 \mathrm{~mol} \mathrm{~L}^{-1}$ was therefore chosen for online FI CPE.

In traditional CPE, addition of $\mathrm{Na}_{2} \mathrm{SO}_{4}$ to the surfactant micellar solution can often reduce the cloud point temperature. Too much $\mathrm{Na}_{2} \mathrm{SO}_{4}$ made the surfactant phase milkywhite, however, which made it more difficult to separate surfactant phase accurately. In this study, the optimum concentration was selected as $0.6 \mathrm{~mol} \mathrm{~L}^{-1}$.

\section{Effect of cotton amount in on-line FI CPE column}

In an on-line FI CPE column the filtering material can entrap larger size surfactant aggregates and let smaller size components pass through, including salt and water molecules, the unretained test compound, surfactant monomer, and surfactant aggregates of smaller size. Therefore, the selection of the filtering material in on-line FI CPE column is important. Huie et al. [9] investigated different filtering materials and found that cotton resulted in the best reproducibility. On the basis of previously reported results $[9,11]$, moreover, an inner diameter of $3 \mathrm{~mm}$ and a length of $3.0 \mathrm{~cm}$ were used for the on-line FI CPE column in this work.

The amount of cotton in collection column was optimized. Results showed that small amounts resulted in low CL intensity because of inefficient collection of surfactant-rich phase whereas larger amounts led to deterioration of the CL signal because of blockage of channels and insufficient washing-out of the retained surfactant-rich phase. In this work, $0.03 \mathrm{~g}$ cotton, leading to a column density of approximately $0.28 \mathrm{mg} \mathrm{mm}^{-3}$, was sufficient for extraction.

\section{Extraction efficiency}

Extraction efficiency is the ratio of the BaP concentration retained in the collection column to the original concentration [19]. As shown in Fig. 2, as sample-loading time was increased from 10 to $140 \mathrm{~s}$, extraction efficiency was gradually decreased from $97.3 \%$ to $38.8 \%$, because cotton sorption efficiency decreased with loading time. When the sample-loading time exceeded $100 \mathrm{~s}$, the extraction efficiency decreased sharply. These results showed that $100 \mathrm{~s}$ sample-loading time almost resulted in adsorption saturation of the cotton column, which was helpful for selecting an appropriate sample-loading time.

In traditional CPE, the extraction efficiency for BaP was estimated by determining the concentration of BaP present in the top layer and the original concentration. The results indicated that the extraction efficiency was ca $86 \%$ for a sample solution containing $0.05 \mu \mathrm{g} \mathrm{mL}^{-1} \mathrm{BaP}$ and $1.0 \%$ Triton X-114. In traditional CPE improvement of the extraction efficiency can be achieved by increasing the concentration of surfactant, although higher surfactant concentrations can result in lower preconcentration factor. For on-line FI CPE, improvement of the extraction 


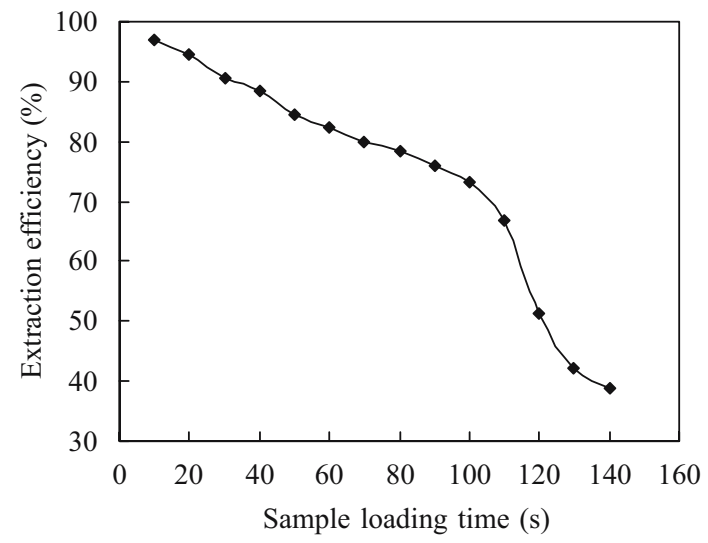

Fig. 2 Effect of sample-loading time on extraction efficiency. The concentrations of Triton $\mathrm{X}-114, \mathrm{Na}_{2} \mathrm{SO}_{4}$, and $\mathrm{BaP}$ were $0.05 \%(v / v)$, $0.8 \mathrm{~mol} \mathrm{~L}^{-1}$, and $0.05 \mu \mathrm{g} \mathrm{mL}^{-1}$, respectively. For a sample-loading time of $90 \mathrm{~s}$ the loading rate (flow rate of aqueous $\mathrm{Na}_{2} \mathrm{SO}_{4}$ solution) was $2.3 \mathrm{~mL} \mathrm{~min}^{-1}$ and the elution rate was $1.5 \mathrm{~mL} \mathrm{~min}^{-1}$. The filter material was $0.03 \mathrm{~g}$ cotton

efficiency can be achieved by changing CPE column length and the amount of cotton packed in the collection column. This was another merit of on-line FI CPE.

\section{Sample-loading time}

To obtain the largest preconcentration factor and greatest extraction efficiency the effect of sample-loading time was examined. From the Fig. 3 we can see that the CL intensity increased with increasing sample-loading time. These results showed the feasibility of achieving higher preconcentration factor by increasing sample-loading time. Considering the extraction efficiency of the collection column and the analytical time, the sample-loading time of $90 \mathrm{~s}$ was chosen as the optimum condition.

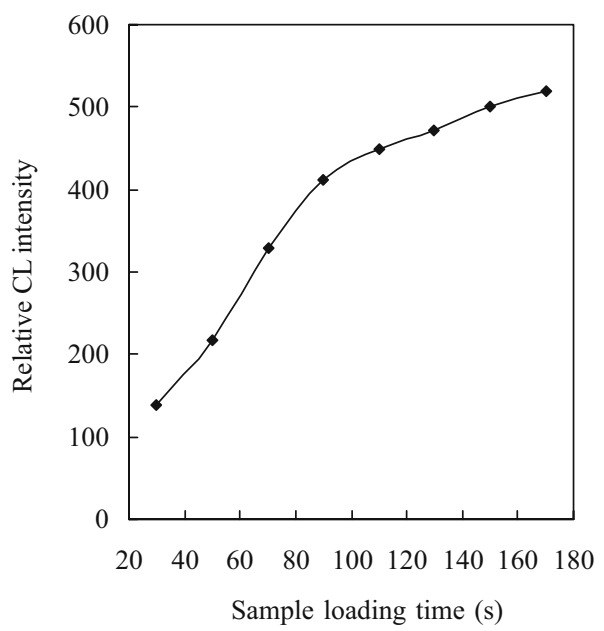

Fig. 3 Effect of sample-loading time on CL intensity. All conditions as for Fig. 2

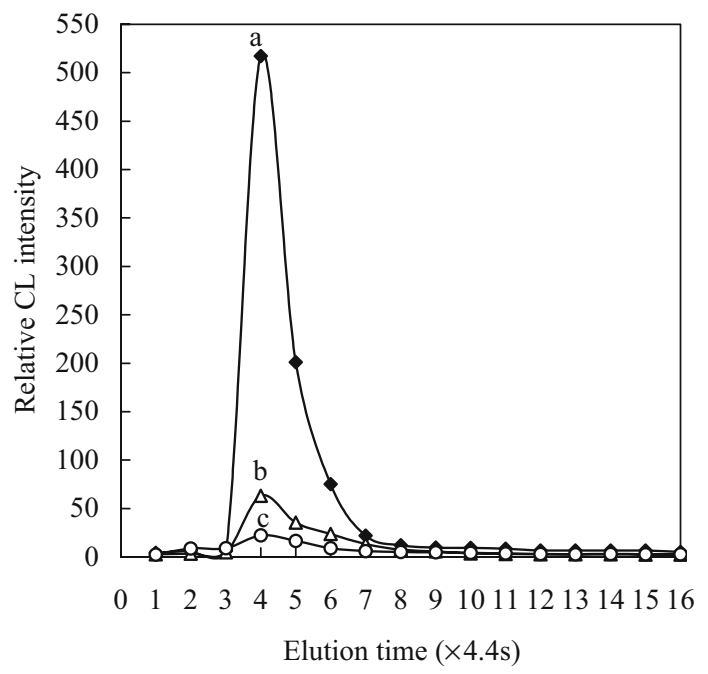

Fig. 4 Three elution curves. Diamonds: $\mathrm{R}_{1}$, sample solution ( $\mathrm{BaP}$ standard and Triton X-114); $\mathrm{R}_{2}, \mathrm{Na}_{2} \mathrm{SO}_{4}$ solution; $\mathrm{R}_{3}$, acetonitrile. Triangles: $\mathrm{R}_{1}$, sample solution (BaP standard), $\mathrm{R}_{2}$, water; $\mathrm{R}_{3}$, acetonitrile; Circles: $\mathrm{R}_{1}$, sample solution (BaP standard and Triton $\mathrm{X}-114$ ); $\mathrm{R}_{2}$, water; $\mathrm{R}_{3}$, acetonitrile; All other conditions as for Fig. 2

\section{Elution curves}

To remove analyte-containing surfactant aggregates within the filter material an appropriate solvent was used for the elution of the analytes and surfactant aggregates from the collection column by switching to the second stage (Fig. 1b). It is well known that CL efficiency can be severely quenched in the presence of water [20]. Therefore, $100 \%$ acetonitrile was used as the eluent.

Three different elution curves were obtained under different conditions, as shown in Fig. 4. When on-line FI CPE was performed (diamonds) it was found that $98 \% \mathrm{BaP}$ could be eluted with $0.66 \mathrm{~mL}$ acetonitrile in $26.4 \mathrm{~s}$. Without surfactant and salting-out agents in on-line FI CPE (triangles) weaker CL signals were observed, because the cotton adsorbed some of the BaP molecules. Furthermore, replacement of $\mathrm{Na}_{2} \mathrm{SO}_{4}$ by $\mathrm{H}_{2} \mathrm{O}$ (circles) resulted in lower maximum $\mathrm{CL}$ intensity, probably because the $\mathrm{BaP}$ molecules can easily enter the Triton X-114 cavity; if CPE did not occur, however, the number of the surfactant aggregates was so low and the cavity so small that the mostly mixed solution of $\mathrm{BaP}$ and Triton X-114 passed through the cotton column. These results clearly showed that concentration of $\mathrm{BaP}$ on the cotton column was mainly a result of CPE.

Table 1 Relative CL intensities for on-line FI CPE and traditional CPE

\begin{tabular}{lcc}
\hline Experiments & \multicolumn{2}{c}{ Relative CL intensity $^{\mathrm{a}}$} \\
\cline { 2 - 3 } & On-line FI CPE & Traditional CPE \\
\hline Original solution & $20.4 \pm 0.1$ & $20.4 \pm 0.1$ \\
Blank solution & $26.7 \pm 1.6$ & $28.3 \pm 3.2$ \\
Concentrated solution & $846.2 \pm 2.8$ & $432.2 \pm 4.7$ \\
\hline
\end{tabular}

${ }^{\mathrm{a}}$ All the results are means from five determinations \pm S.D. 


\section{Preconcentration factor}

Table 1 shows the relative CL intensities for on-line and traditional CPE. It was found that the relative CL intensity of on-line FI CPE was much higher than that of traditional $\mathrm{CPE}$. In this study the preconcentration factors for on-line FI CPE and traditional CPE were 40.2 and 19.8, respectively. The sample volume used in on-line FI CPE, $3.45 \mathrm{~mL}$, was, moreover, a factor of 7.4 less than that used in traditional CPE. The efficiency of concentration of other $\mathrm{PAH}$, for example BkF and Bper, was also compared. The results showed that the preconcentration factors for these PAH in on-line FI CPE were much higher than in traditional CPE - the values were 38.5 and 41.3, respectively.

\section{Analytical time and some technical aspects}

In traditional CPE, separation of the surfactant aggregates from the bulk aqueous solution into two distinct phases is usually accomplished by allowing the solution to stand for a given time (e.g. $30 \mathrm{~min}$ ) and subsequently by using centrifugation to hasten this phase-separation process. The equilibration and centrifugation procedures usually require half an hour or longer, and inadequate equilibration and centrifugation can lead to reduced extraction efficiency. Importantly, after separation of the two phases, manual removal of the surfactant-rich phase with a pipette can cause undesirable experimental error.

In on-line FI CPE, without long equilibration and centrifugation procedures, the entire CPE process can be completed in three minutes by using the flow injection channels to mix the solutions and using a man-made collection column packed with cotton to separate the surfactant-rich phase from the bulk aqueous solution. In this work, when the FIA system was switched to the elution stage (Fig. 1b), approximately 98\% BaP could be effectively eluted using $0.66 \mathrm{~mL}$ acetonitrile as eluent in 26.4 s. Therefore, on-line FI CPE can achieve a higher preconcentration factor and greater sample throughput.

\section{Conclusions}

In the work discussed in this paper, on-line and traditional $\mathrm{CPE}$ were compared in detail. The performance of on-line
FI CPE has been systemically discussed. The results indicate that the analytical capability of on-line FI CPE, especially in terms of preconcentration factor, extraction efficiency, and analysis time, are a considerable improvement over the traditional CPE method. On the basis of this behavior it should be possible to develop on-line FI CPE preconcentration coupled with separation techniques such as HPLC and CE.

Acknowledgements The authors gratefully acknowledge financial support from the National Science Fund for Distinguished Young Scholars of China (No. 20125514), the National Natural Science Foundation of China (No. 20437020, 50273046), NSFC-JSPS Joint Research Project (No. 20511140134), and the Major Research Program of Chinese Academy of Sciences (KZCX3-SW-432).

\section{References}

1. Gu T, Galera-Gómez PA (1995) Colloids Surf A 104:307

2. Saitoh T, Hinze WL (1991) Anal Chem 63:2520

3. Lu C, Lin J-M (2004) Acta Phys-Chim Sin 20:974

4. Delgado B, Pino V, Ayala JH, González V, Afonso AM (2004) Anal Chim Acta 518:165

5. Ferrera ZS, Sanz CP, Santana CM, Rodríguez JJS (2004) Trends Anal Chem 23:479

6. Tani H, Kamidate T, Watanabe H (1997) J Chromatogr A 780:229

7. Wang J, Hansen EH (2003) Trends in Anal Chem 22:225

8. Laespada MEF, Pavón JLP, Cordero BM (1993) Analyst 118:209

9. Fang Q, Du M, Huie CW (2001) Anal Chem 73:3502

10. Paleologos EK, Vlessidis AG, Karayannis MI, Evmiridis NP (2003) Anal Chim Acta 477:223

11. Garrido M, Di Nezio MS, Lisa AG, Palomeque M, Band BSF (2004) Anal Chim Acta 502:173

12. Lu C, Lin J-M, Huie CW, Yamada M (2004) Anal Chim Acta 510:29

13. Lin J-M, Yamada M (1999) Anal Chem 71:1760

14. Pino V, Ayala JH, Afonso AM, González V (2002) J Chromatogr A 949:291

15. Lin J-M, Yamada M (2003) Trends Anal Chem 22:99

16. Dan N, Lau ML, Grayeski ML (1991) Anal Chem 63:1766

17. Horvath WJ, Huie CW (1993) Talanta 40:1385

18. Paleologos EK, Stalikas CD, Karayanni SMT, Pilidis GA, Karayannis MI (2000) J Anal At Spectrom 15:287

19. Horvath WJ, Huie CW (1992) Talanta 39:487

20. Imaizumi N, Hayakawa K, Miyazaki M (1989) Analyst 114:161 